\title{
Research on Design of Ethernet Communication Interface
}

\section{Based on ARM}

\author{
CHEN Xuechang ${ }^{1, a}$ \\ ${ }^{1}$.Institute of Applied Electronics , Chongqing College of Electronic Engineering; Chongqing 401331, China \\ a.Email: pcboy2004@126.com
}

\section{Keywords: ARM Protocol, Communication Interface, Serial Communication}

\begin{abstract}
With the expansion of control system and the development of bus technology, the data collection and transmission technology put forward higher requirements. With the development and popularization of Ethernet technology, the serial data of these devices need to be transmitted through the network, so it is necessary to seek a solution to realize the technical innovation. In this paper, serial communication and Ethernet communication based on TCP / IP protocol are researched and analyzed respectively. A serial communication system based on ARM processor and Ethernet communication system is designed to realize the multi-channel serial data in the embedded system. In this paper, the overall design of the system and function module division, and details of the ARM processor-based Ethernet communication interface and data transmission interface circuit design. In the software design, the system startup code, serial communication protocol, serial port driver and multi-serial port and the network port between the two-way data transmission.
\end{abstract}

\section{Theoretical Introduction of ARM}

ARM processor is based on the RISC processor family, the processor core series for its excellent low power, low cost, multi-family, data transmission and processing speed and become a variety of digital mobile communication terminals and network products of choice. The processor mode can be switched by software control, or by an external interrupt or exception handler. Most user programs run in user mode. The future of the Internet world is TCP / IP, TCP / IP combination is the trend with high practical value.

ARM processor three features include: less power consumption, 16-bit / 32-bit dual instruction set and a large number of partners. ARM (32-bit) dual instruction set can be very compatible with 8/16-bit devices; extensive use of registers, the instruction execution speed is faster, low power consumption, low cost, high performance; most data operations are completed in the register; addressing flexible and simple way, the implementation of high efficiency; instruction length is fixed ${ }^{[1]}$.

Ethernet work in the data link layer, through different transmission media and conflict detection methods, for the network layer, data link layer to provide efficient services. The ARM processor has a total of 37 registers: 31 general-purpose registers, including the program counter ((PC): 6 status registers, the registers are 32-bit. When $\mathrm{j}$ before the program status register can be in any processor mode, which contains condition flags, interrupt disable bits, current processor mode flags, and other control and statuses. When processor mode switching is required, the application can generate exception handling and handle the processor during exception processing mode switch, this architecture allows the operating system to control the resources of the entire system.

\section{System Design of Ethernet Communication Interface}

System reset module provides S3C2440 start signal, it is the beginning of the whole system operation. S3C2440 has two asynchronous reset signals: nRESET and nTRST. Such as their valid signals, the system reset will be internally generated. All internal registers except the RTC data and match registers will be reset. SP706 can monitor UP and digital systems in the power supply and battery operating status. It includes a watchdog timer, an UP reset module, a power fail comparator, and a manual reset input module ${ }^{[2]}$. Fig. 1 shows system reset circuit. 


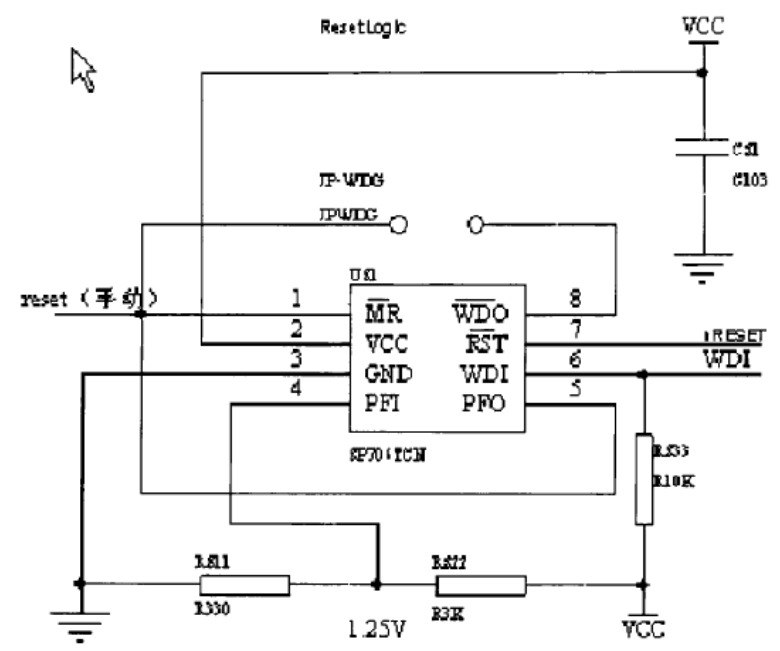

Fig. 1.The system reset circuit

In the system, the need to use SV and 3.3V DC power supply, which, S3C2440 and some peripheral devices need 3.3V power supply, and some devices need SV power supply, to simplify the system power supply circuit design requires the entire system input voltage The LT1083 converter for high quality SV DC regulated power supplies can perform SV to $3.3 \mathrm{~V}$ conversion. The system reset circuit composed of SP706 is shown in Figure 4-2. It has the following functions: high-precision voltage monitoring, independent watchdog timer-overflow period 1.6s, manual reset, power failure and low battery warning monitor.

Memory SDRAM module has the advantages of large storage capacity per unit space and low cost, and has been widely used in various embedded systems. SDRAM memory cell can be understood as a capacitor, always inclined to discharge, in order to avoid data loss, you must regularly refresh (charge). Therefore, to use SDRAM in the system, requires the microprocessor to refresh the control logic, or in the system to add another refresh control logic. SDRAM in the system is mainly used for the program running space, data and stack area. When the system starts, the CPU first reads the startup code from the reset address 0x0. After the initialization of the system, the program code should be transferred to the SDRAM to improve the system running speed ${ }^{[3]}$.

\section{Hardware Design Module}

In this design, the ARM microprocessor's role is to collect the data collected by the voltage acquisition unit after the A / D conversion through the Ethernet unit to the PC client, PWM, LCD and USB modules reserved for functional expansion. Fig.2 shows hardware design of the system.

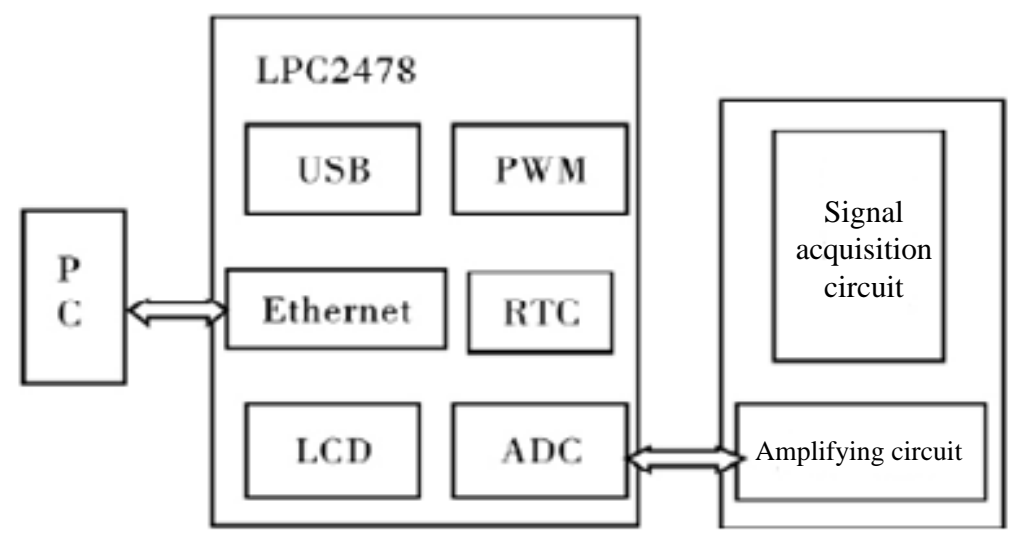

Fig. 2. The hardware design of the system

Protocol conversion is the key and difficult technology of network interconnection. At present, most of the interconnection in the network layer or network layer to expand, and therefore must be the following layers of the interconnection layer protocol layer up conversion. This conversion 
method is based on the basic principles of protocol layering, that is, low-level support for high-level, high-level call low-level, low-level disconnection, high-level connections will be disconnected, but high-level disconnect will not affect the lower. The data link layer and the physical layer are defined by the CAN protocol: the ARM data gateway has the physical layer, the data link layer and the application layer, and its application layer is also the information and data exchange layer of the network and CAN, the ARM The micro-controller interprets and forwards the data of the two different protocols at this layer. Ethernet runs TCP / IP protocol, it have the application layer, transport layer, network layer Ethernet data link layer and physical layer CAN bus with the application layer, data link layer and physical layer.

In this design, ARM gateway is designed as a transparent data gateway. That is, in the Ethernet application layer to build and resolve the complete CAN protocol packets. ARM IP protocol design and implementation of TCP / IP protocol suite is the most core of the agreement. All TCP, UDP, ICMP, and IGMP data is transmitted in IP datagram format. OIP provides unreliable, connectionless datagram delivery. As a simple data transmission of the basic networking model, Ethernet was widely used in various occasions. The design of the IP protocol is a special application environment for the reasonable simplification ${ }^{[4]}$.

\section{Communication Interface Module}

The Ethernet interface circuit of the system should be indispensable, but it is also relatively complicated at the same time. From the hardware point of view, the Ethernet interface circuit mainly by the MAC controller and physical layer interface composed of two parts, the current common Ethernet interface chip internal structure also includes these two parts. It provides 10M / $100 \mathrm{MBps}$ Ethernet access in half-duplex or full-duplex mode. In half-duplex mode, the controller supports CSMA / CD protocol, in full-duplex mode to support IEEE802.3 MAC control layer protocol. Fig.3 shows Network isolation transformer and RJ45 connection circuit.

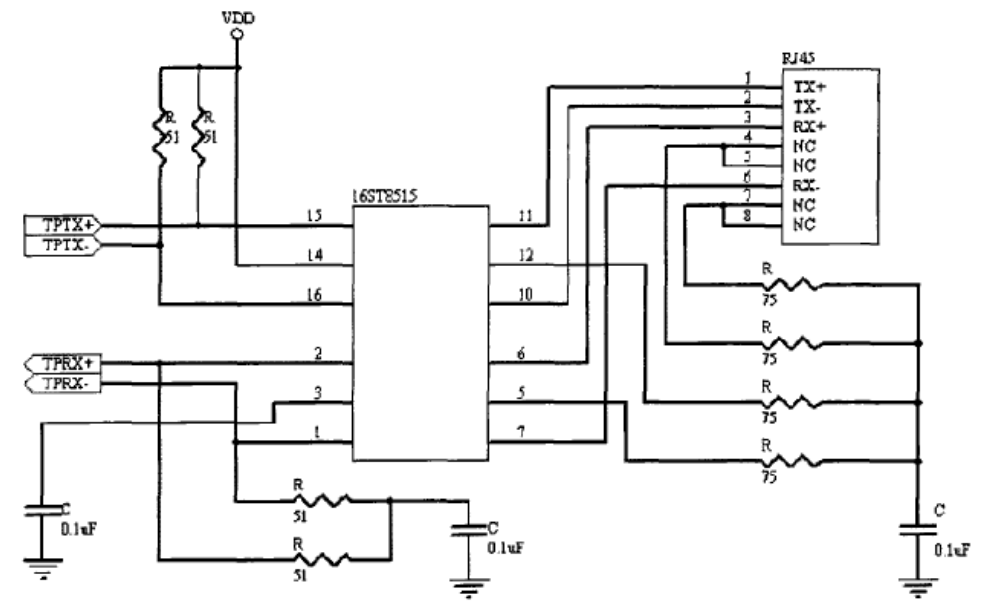

Fig. 3. Network isolation transformer and RJ45 connection circuit

Through a DART transmission signal, then achieve a transceiver. Transceiver uses balanced transmission and differential reception, that is, at the sending end, the driver converts the TTL level to a differential signal output. At the receiving end, the receiver changes the differential signal to TTL level, thus suppressing common mode interference. On the receiver with high sensitivity, can monitor the voltage as low as 200mv, so the data transmission up to 1000 meters away. Commonly used single-port 10M / 100MBps high-speed Ethernet physical layer interface devices are RTL8201, DM9161, etc., provide MII interface and the traditional 7-wire network interface can be easily and S3C2440 interface. Ethernet physical layer interface device main functions generally include: physical coding sublayer, physical media accessories, twisted pair physical media sublayer, encoder, decoder and twisted pair media access unit ${ }^{[5]}$.

Signal transmission and receiving end through the network isolation transformer and RJ45 interface access to transmission media. When connected to different Ethernet, you can use a 
computer program using the appropriate settings via RS-232 of the gateway, IP address, subnet mask and other parameters, is transmitted to the system introduced at work. After the hardware platform of the system is successfully built, the application program based on ARM development board is written. The program works on the embedded system, and can send the sensor data to the client through Ethernet. The client PC can access the incoming data through the browser.

\section{Conclusions}

In recent years, with the development and popularization of Ethernet technology, open Internet technologies have been widely used in various fields. ARM processor because of its high performance, low cost, low power consumption, has become the most widely embedded processor. Ethernet speed advantage, low port price and superior performance, for the transmission of large quantity, high real-time requirements of industrial control production areas, which has a great attraction. Based on the embedded serial communication technology of ARM, the communication module is a system with high technical content integrated with computer digital control, ARM, serial communication and Ethernet communication. Based on ARM embedded Ethernet interface implementation will have a good application prospects.

\section{References}

[1] CAVENDISH D. Operation, administration, and maintenance of Ethernet services in wick area networks [J].Communications Magazine, IEEE, 2008, 42 C3):75-79.

[2] Tang H R, Dong W, The outlook of fieldbus networks technology [J]. Monitoring and Control Technologies, 2010.19 (2):14.

[3] FELSER M. Standardization of industrial Ethernet the next battlefield. IEEE Symposium on World Factory Communication Systems, 2014(10):413-421.

[4] ZhouYongqin, Wang Yudong, Zhou Meilian. The Research and Realization for Passenger Car CAN Bus [J]. IFOST, 2014, 10: 34-47.

[5] Li DZ, Wang CA. The implementation of CAN Ethernet communication system on the missile simulation and detection platform. IEEE 15th International Conference, Kun ming, 2014, 11. 